\title{
Decreased Stress Responsivity of Central and Peripheral Catecholaminergic Systems in Aged 344/N Fischer Rats
}

\author{
Giovanni Cizza, ${ }^{\natural \# ~ K a r e l ~ P a c a k, ~}{ }^{\ddagger}$ Richard Kvetnansky, \\ Koki Fukuhara, ${ }^{\ddagger}$ Ettore Bergamini, ${ }^{\#}$ Irwin J. Kopin, ${ }^{\ddagger}$ Marc R. Blackman, ${ }^{\star \star}$ George P. Chrousos, ${ }^{\natural}$ and Philip W. Gold ${ }^{\star}$ \\ ${ }^{*}$ Clinical Neuroendocrinology Branch, National Institute of Mental Health; ${ }^{\ddagger}$ Clinical Neuroscience Branch, National Institute of \\ Neurological Disorders and Stroke; ${ }^{\circledR}$ Laboratory of Cell Biology, National Institute of Mental Health, Bethesda, Maryland 20892; \\ "Institute of Experimental Endocrinology, Bratislava, Slovakia; "Developmental Endocrinology Branch, National Institute of Child Health \\ and Human Development; **Departments of Medicine, Francis Scott Key Medical Center and the Johns Hopkins University School of \\ Medicine, Baltimore, Maryland 21224; and ${ }^{\ddagger \ddagger}$ Istituto di Patologia Generale, Universita’ di Pisa, Pisa 56100, Italy
}

\begin{abstract}
We investigated the effects of stress on central and peripheral sympatho-adrenal and sympatho-neural functions in healthy, intact young (3-4 mo) and aged (24 mo) male Fischer 344/N rats.

Extracellular fluid (ECF) levels of the catecholamines norepinephrine (NE), dihydroxyphenylglycol (DHPG), methoxyhydroxyphenylglycol (MHPG), and dihydroxyphenylacetic acid (DOPAC) were obtained by microdialysis in the paraventricular nucleus (PVN) of the hypothalamus at baseline and during immobilization (IMMO). The baseline levels of these substances were similar in both age groups, and their concentrations increased significantly in response to IMMO. The IMMO-induced increases of NE and MHPG, however, were significantly smaller in old than in young rats.
\end{abstract}

Plasma levels of the catecholamines NE, DHPG, MHPG, DOPAC, dihydroxyphenylalanine (DOPA), epinephrine (EPI), dopamine (DA), and HVA were also determined in young and old rats during IMMO. Basal levels of these substances were significantly higher in old than in young rats. The magnitude of the IMMO-induced increases in the majority of these compounds however, was significantly smaller in old than in young rats.

We conclude that, at the basal state, aging in the Fischer rat is associated with normal PVN ECF, but high plasma catecholamine levels; at stress state, however, old rats have substantially lesser activation of their central and peripheral catecholaminergic systems than young rats. (J. Clin. Invest. 1995. 95:1217-1224.) Key words: microdialysis • PVN • locus ceruleus • tyrosine hydroxylase $・$ catecholamine-metabolites

\section{Introduction}

With advancing age, many species exhibit a decreased capacity to maintain homeostasis after exposure to various stressors (1). Stress responses result from coordinated activation of several

Part of these data were presented at the 74th Annual Meeting of the Endocrine Society, San Antonio, TX, June 24-27 1992, and at the 7th International Catecholamine Symposium, Amsterdam, June 22-26 1992.

Address correspondence to Giovanni Cizza M.D., Ph.D., Clinical Neuroendocrinology Branch, NIMH, Bldg. 10, Rm. 3S-225, 9000 Rockville Pike, Bethesda, MD 20892. FAX: 301-402-1561.

Received for publication 2 May 1994 and in revised form 11 November 1994.

The Journal of Clinical Investigation, Inc.

Volume 95, March 1995, 1217-1224 effector systems, including, importantly, the sympathoneural and sympathoadrenal systems and the hypothalamic-pituitaryadrenal (HPA) ${ }^{1}$ axis (reviewed in reference 2 ). The principal central nervous system (CNS) components of the stress response include catecholaminergic areas located in the brain stem, and corticotropin-releasing-hormone ( $\mathrm{CRH}$ ), which regulate the peripheral sympatho-neural system and the pituitaryadrenal axis, respectively (2). These two systems are anatomically and functionally connected to each other in a positive, reverberatory circuit, which is activated during stress (2).

Aging complexly affects the sympathoneural and sympathoadrenal systems and the HPA axis (1). In rats, age-related changes in central noradrenergic functions, such as decreased tissue catecholaminergic concentration of norepinephrine (NE) (3), delayed recovery in NE turnover after immobilization (IMMO) stress (4), and impaired alpha-adrenergic receptor synthesis (5), appear to be functionally related to memory deficits and impaired acquisition of novel tasks (6); caloric restriction, which generally extends the life span of rodents, leads to a diminution of hypothalamic catecholamine levels (7).

Aging-associated diminutions in various parameters of activity of locus ceruleus ( $\mathrm{LC}$ ) neurons, the major source of brain catecholamines, such as reduced electrical activity (8) and neuronal loss (9), have been reported in rodents, in normal humans (10), as well as in depression (10) and in various degenerative diseases such as Alzheimer's and Parkinson's diseases (11). The decrements in LC neuronal function, however, appear to be species- and strain-specific, as no changes in the number of noradrenergic neurons in the $L C$ of $F / 344 \mathrm{~N}$ rats have been observed up to 32 mo of age (12).

In aged rats and humans, basal plasma levels of NE are usually increased $(13,14)$, whereas basal 1-dihydroxyphenylalanine (DOPA) levels are significantly decreased with age (15). Activities of several enzymes involved in the catecholamine synthetic pathway, such as tyrosine hydroxylase (TH), dopamine- $\beta$-hydroxylase, and phenylethanolamine- $N$-methyltransferase, are also increased in the adrenal medulla of older rats $(16,17)$. Adrenergic receptors control NE turnover by modulating the release of NE from noradrenergic terminals; a number of age-related alterations in adrenergic receptor and post-receptor functions have also been reported, such as decreases in the

\footnotetext{
1. Abbreviations used in this paper: AUC, area under the curve; $\mathrm{CRH}$, corticotropin-releasing-hormone: DA, dopamine; DHPG, dihydroxyphenylglycol; DOPA. dihydroxyphenylalanine; DOPAC, dihydroxyphenylacetic acid; ECF, extracellular fluid; EPI, epinephrine; IMMO, immobilization; LC, locus ceruleus; MHPG, methoxyhydroxyphenylglycol; NE, norepinephrine; PVN, paraventricular nucleus; TH, tyrosine hydroxylase.
} 
number of adrenergic receptors in several tissues, impairments in the capacity to develop either desensitization or supersensitivity, and diminished intracellular calcium flux (reviewed in references 18 and 19).

In the present study, we sought to characterize age-associated changes in central and peripheral catecholaminergic activity of young and old male 344/N Fischer rats, a well characterized strain for aging studies, under basal conditions and during acute IMMO, a stressor known to activate both catecholaminergic systems and the HPA axis $(20,21)$. Our results suggest that the magnitude of IMMO-induced activation of central and peripheral catecholaminergic systems is reduced in old versus young rats.

\section{Methods}

\begin{abstract}
Animals
Intact young (3-4 mo) and old (24 mo) virgin male Fischer 344/N (F $344 / N$ ) rats were purchased from Harlan-Sprague Dawley, Inc. (Indianapolis, IN). Before initiation of experimental procedures, rats were housed (2-3 per cage) for at least one week, in cages equipped with a laminar flow unit and air filters. Animals had free access to food and water, and were under constant temperature, humidity, and light conditions with a 12-h light, 12-h dark cycle (lights on at $0600 \mathrm{~h}$ ). Animals not in good health were excluded from the study. Separate groups of rats were used for experiments involving microdialysis, in situ hybridization, and hormone determinations in plasma. The procedures employed in the present study were approved by the NICHD and the NINDS Animal Care and Use Committees.
\end{abstract}

\section{Immobilization protocol}

On the day of the experiment, rats (6-9 per group) were immobilized, as previously described (21), in a prone position by inserting their heads through steel wire loops fixed on a board, and by fastening their limbs to four metal strips with adhesive tape. Using this method, different groups of rats were simultaneously immobilized for $5,30,60$, or $120 \mathrm{~min}$ and were sacrificed either immediately after the immobilization period or after a $2 \mathrm{~h}$ recovery period.

\section{In vivo assessment of extra-cellular fluid levels of norepinephrine and its metabolites by microdialysis in the hypothalamic paraventricular nucleus}

Probe insertion. As previously described (20), rats were anesthesized with pentobarbital $(50 \mathrm{mg} / \mathrm{kg}$ BW i.p.) and placed in a stereotaxic apparatus (David Kopf Instruments, Tujunga, CA) with the incisor bar $3.2 \mathrm{~mm}$ below the interaural line. The skull was then exposed, and a small hole was drilled over the right paraventricular nucleus (PVN). A small microdialysis probe $(1 \times 0.5 \mathrm{~mm}$, with $1.0-\mathrm{mm}$-long permeable polycarbonate-ether dialysis membrane at its tip; mol wt cutoff: 20,000 D; BAS/Carnegie Medicin, West Lafayette, IN) was placed according to the following coordinates relative to the bregma: young rats anterior: caudal, $1.9 \mathrm{~mm}$; lateral, $0.5 \mathrm{~mm}$; vertical, $8.5 \mathrm{~mm}$; old rats: anterior caudal, $1.9 \mathrm{~mm}$; lateral, $0.5 \mathrm{~mm}$; vertical, $8.8 \mathrm{~mm}$. (22) (Fig. 1). Samples were taken from the extracellular fluid from an area of $\sim 0.3-0.5$ $\mathrm{mm}$ radius around the membranous tip of the probe. The PVN in the rat is a relatively small group of cells with the following dimensions: maximal medial-lateral extension $0.8-1.0 \mathrm{~mm}$; rostro-caudal extension 0.6-0.8 $\mathrm{mm}$; ventro-lateral extension (maximum) $0.4-0.7 \mathrm{~mm}$. The center of the PVN is equally distant from the third ventricle and the fornix medio-laterally. Our criterium of acceptance in the histological controls was that the probes would be located centrally into the PVN. The implanted microdialysis probe was anchored to the skull with three stainless steel skull screws and fixed with acrylic dental cement. Immediately after surgery, the probe was connected to a microinfusion pump (CMA 100; BAS/Carnegie Medicin, West Lafayette, IN), and artificial cerebrospinal fluid ( $189 \mathrm{mM} \mathrm{NaCl}, 3.9 \mathrm{mM} \mathrm{KCl}$, and $3.37 \mathrm{mM} \mathrm{CaCl}$, $\mathrm{pH}$ 6.3) was continuously perfused at a rate of $1.0 \mu \mathrm{l} / \mathrm{min}$. After surgery, rats recovered for 20-24 h before experiment in cylindrical plexiglas cages, one per cage, with food and water freely available.

Microdialysis. All experiments began between 08:00 and 09:00 h. Young and old rats were simultaneously immobilized for $120 \mathrm{~min}$, and were then returned to their home cages and left undisturbed for another $120 \mathrm{~min}$. Dialysate was collected at 30-min intervals, $90 \mathrm{~min}$ before, $120 \mathrm{~min}$ during, and $120 \mathrm{~min}$ after the IMMO procedure. Thus, 11 samples ( 3 samples before, 4 samples during, and 4 samples after the termination of the IMMO procedure) were collected during a total period of $330 \mathrm{~min}$. The samples were collected in vials containing $5 \mu \mathrm{l}$ of 0.2 $\mathrm{N}$ acetic acid, and immediately frozen in dry ice. At the end of the experiment, the animals were decapitated and the brains were removed and stored in $10 \%$ formalin solution for subsequent histological verification of probe position. In 6 young and 7 old rats the probe was positioned correctly into the PVN, and data generated from these animals were used. Rats in which the probe had not been correctly placed according to the criteria described above were excluded from the study.

\section{Assessment by in situ hybridization histochemistry of} tyrosine hydroxylase mRNA levels in the locus coeruleus

Fresh frozen sections (15- $\mu$ m-thick) were cut coronally at the level of the LC ( $-9.7 \mathrm{~mm}$ relative to bregma). Sections were thaw-mounted onto gelatin-coated slides, dried, and stored at $-40^{\circ} \mathrm{C}$ before being processed. Synthetic 48-base oligodeoxyribonucleotide probes were directed against TH bases 1441-1488 (23). The in situ hybridization histochemistry was performed as previously described (24). Slides and ${ }^{35} \mathrm{~S}$-impregnated brain paste standards of known radioactivity and wet weight were placed in $\mathrm{x}$-ray cassettes, apposed to film (Hyperfilm$\beta \mathrm{Max}$, Amersham, Arlington Heights, IL) for $5 \mathrm{~d}$ and developed (D19; Eastman Kodak Co., Rochester, NY) for $5 \mathrm{~min}$ at $20^{\circ} \mathrm{C}$.

\section{Data analysis}

Autoradiographic film images of brain sections and ${ }^{35} \mathrm{~S}$-standards were analyzed densitometrically using a computer-based image analysis system with IMAGE software (24). A second-order polynomial calibration curve was constructed using the transmittance values of ${ }^{35} \mathrm{~S}$-brain paste standards containing known amount of radioactivity. Transmittance measurements for TH probe were made on four consecutive sections, which were matched for anatomical level. The average value for each rat per group was used to calculate group means $(n=6-7)$. Transmittance measurements were converted to disintegration per $\mathrm{min} / \mathrm{mg}$ of wet weight of tissue using the calibration curve.

\section{Tail artery cannulation procedure}

Details of the cannulation procedure have been described (25). Briefly, animals were anesthetized, and a polyethylene cannula (PE-50; Clay Adams, Pasippany, NJ) was inserted into the ventral caudal artery. The cannula was tunneled under the skin and exited subcutaneously at the back of the neck. After surgery, animals were housed separately with food and water freely available.

To determine $(a)$ whether $24 \mathrm{~h}$ of recovery from surgery was enough to allow plasma catecholamine levels to return to baseline values, and (b) whether old rats needed more time to recover from surgery, rats (young $n=9$; old $n=8$ ) were cannulated as previously described (25), samples were collected at $0800 \mathrm{~h} ., 24 \mathrm{~h}$, and $48 \mathrm{~h}$ after the cannulation, and plasma NE levels were measured. NE levels (mean \pm SEM) were similar at 24 and $48 \mathrm{~h}$ after cannulation in both age groups; at both time points NE levels were significantly higher in old vs. young rats ( $24 \mathrm{~h}$ : young, $343 \pm 42 \mathrm{pg} / \mathrm{ml}$; old, $1107 \pm 263 \mathrm{pg} /$ $\mathrm{ml} ; 48 \mathrm{~h}$ : young, $406 \pm 41 \mathrm{pg} / \mathrm{ml}$; old, $1528 \pm 262 \mathrm{pg} / \mathrm{ml} ; P \leq 0.05$ ). Because plasma levels had returned to baseline by $24 \mathrm{~h}$ and remained stable at $48 \mathrm{~h}$, a $24 \mathrm{~h}$ recovery period was used in the subsequent experiment. On the day of the final experiment, rats $(n=7 /$ group) were simultaneously immobilized, between 0800 and $1200 \mathrm{~h}$ blood samples (400-450 $\mu \mathrm{l}$ ) were collected before (baseline) and after 5, 30, 60, and $120 \mathrm{~min}$ of IMMO. Thus, a volume of $2-2.25 \mathrm{ml}$ of blood was withdrawn from each animal. A volume of saline equal to the sample volume was returned immediately to each rat. This amount of blood can be collected without stimulating a reflex activation of the catechol- 


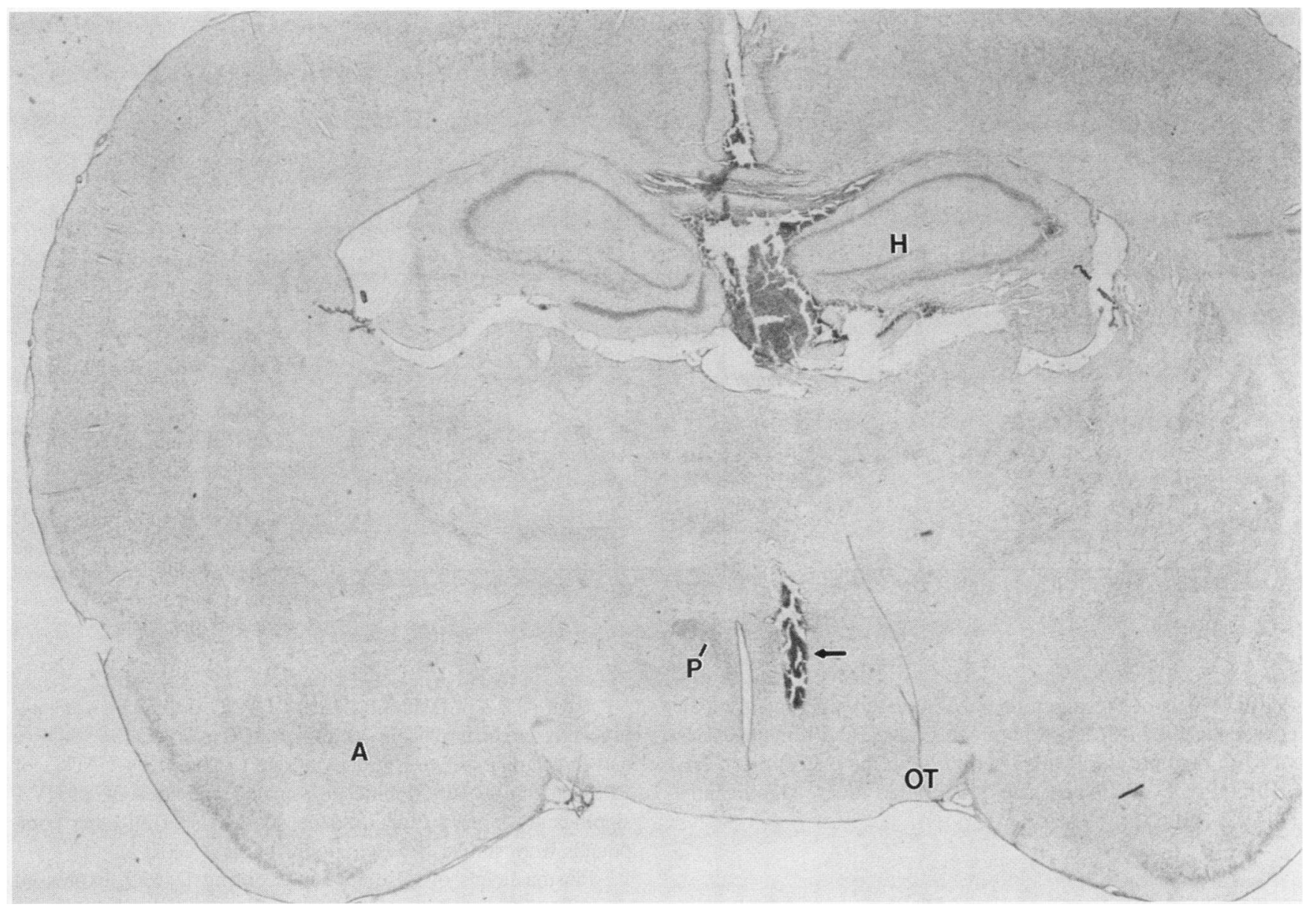

Figure 1. Coronal slice showing implantation of the dialysis probe in the right paraventricular nucleus. $F$, fornix; OT, optic tract.

aminergic system (26). This procedure enabled us to obtain repeated blood samples from conscious animals.

\section{Assays}

Levels of all compounds in microdialysates and plasma were assayed by reversed phase liquid chromatography with electrochemical detection following partial purification by adsorption onto alumina (25). Levels of methoxyhydroxyphenylglycol (MHPG) in the microdialysate were measured in each alumina supernatant (20).

\section{Statistical analyses}

All data are expressed as the means \pm SEM. Data analysis was performed on a Macintosh IIsi computer using SUPERANOVA software (Abacus Concepts Inc., Berkeley, CA). The effects of IMMO on the two age groups in levels of $\mathrm{NE}$ and its metabolites were analyzed by repeated measures analysis of variance, with time of sampling as a within subjects factor, and age as a between subjects factor. To analyze these results further, the total area under the curve (AUC) of all compounds before, during, and after IMMO was calculated by integration of hormone levels in conventional units, and time of testing in minutes (24). The net AUC was also calculated for plasma catecholamines as difference between total AUC and basal AUC (baseline times length of testing) (24), and comparisons were then performed using two-way analysis of variance, followed by Scheffe's test. Comparisons between age groups of TH mRNA levels, at basal conditions and after IMMO were also performed using two-way analysis of variance, followed by Scheffe's test. Significance was accepted at $P<0.05$.

\section{Results}

In vivo assessment of extracellular fluid levels of NE, MHPG, $D H P G$, and DOPAC in the paraventricular nucleus. In both age groups, resting levels of NE, MHPG, dihydroxyphenylglycol (DHPG), and dihydroxyphenylacetic acid (DOPAC) were similar, and IMMO stimulated significant increases of all four compounds (Fig 2). NE levels rapidly increased during IMMO, followed by increases in MHPG, DHPG, and DOPAC. After IMMO, levels of all four compounds decreased similarly in both age groups, but remained greater than the corresponding pre-IMMO levels. Pre-IMMO AUCs of NE, MHPG, DHPG, and DOPAC, were similar between age groups (Fig. 3). During IMMO, the AUCs of NE and MHPG were significantly lower in old vs young rats; there was a nonsignificant $(P<0.06)$ trend towards a lower AUC of DHPG in old rats, whereas the AUCs for DOPAC did not vary with age. After IMMO the AUCs of all catecholamines were lower in old vs young rats, although the age difference was significant only for MHPG. In both age groups, AUCs of all catecholamines were significantly greater after versus before IMMO.

TH mRNA levels in the locus coeruleus. Baseline levels of TH mRNA (dpm/mg wet weight) were similar in young $(795 \pm 85)$ and old $(757 \pm 52)$ rats. Although IMMO produced significant increases in TH mRNA levels in both groups (young, $1554 \pm 78$; old, 1715 \pm 48 ), the increases were not different between age groups (Fig. 4).

Plasma levels of catecholamines and their metabolites in plasma during IMMO. At baseline, levels of EPI, DHPG, MHPG, DOPA, DA, DOPAC, and HVA were all significantly higher in old versus young rats, whereas there was a nonsignificant $(P<0.06)$ trend to higher NE levels with age (Table I). 

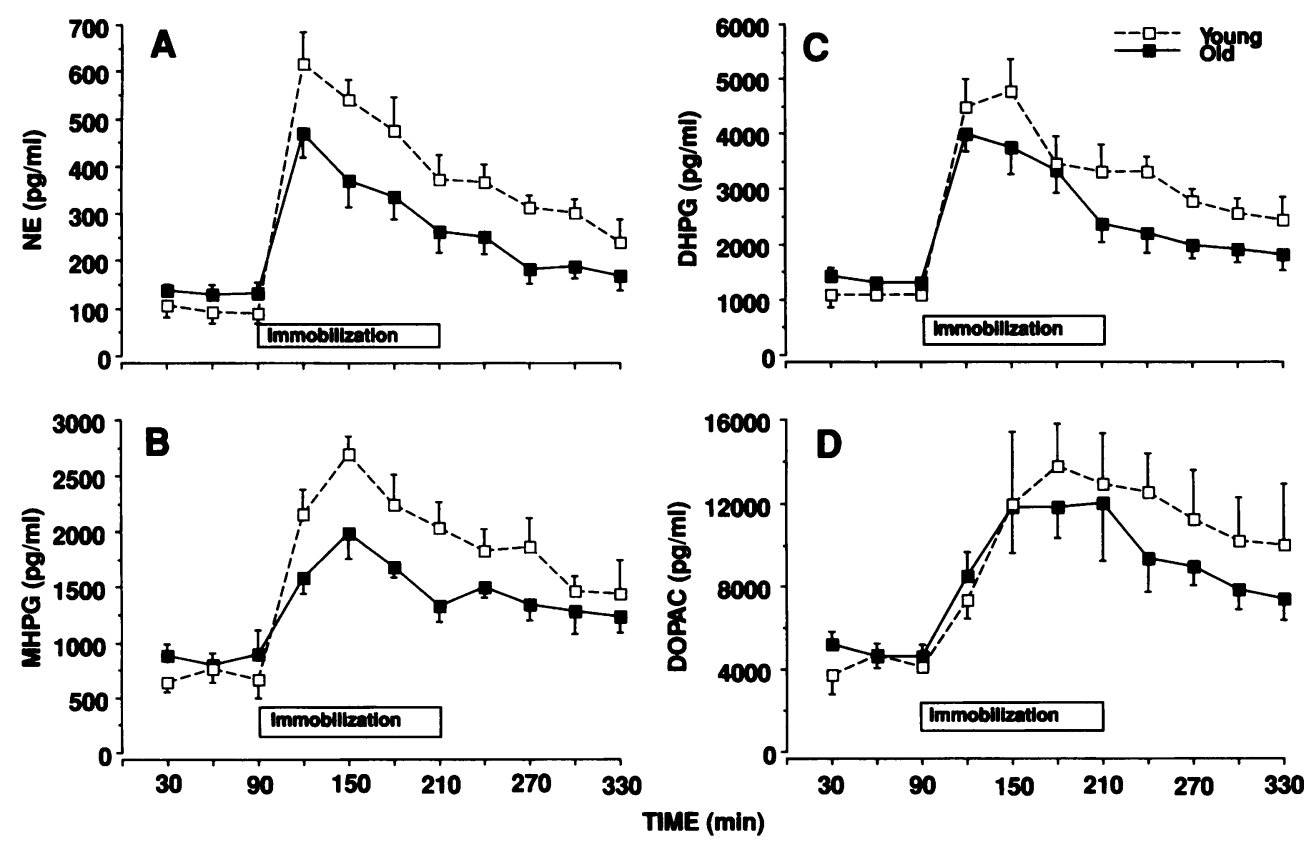

Figure 2. Microdialysate concentration in the paraventricular nucleus of the hypothalamus of $\mathrm{NE}$ $(A), \operatorname{MHPG}(B)$, DHPG $(C)$, and DOPAC $(D)$ in young (3-4-moold) and old (24-mo-old) male Fischer $344 / \mathrm{N}$ rats, before, during, and after immobilization. Each time-point represents the mean \pm SEM of samples of six (young) or seven (old) rats. Resting levels were similar in both age groups; immobilization increased significantly $(P<0.0001)$ levels of all four compounds.
Plasma levels of these compounds increased significantly as a consequence of IMMO in both age groups, and remained elevated throughout the IMMO period, with the exception of NE and DOPA levels, which in old rats failed to rise significantly (Figs. 5 and 6).
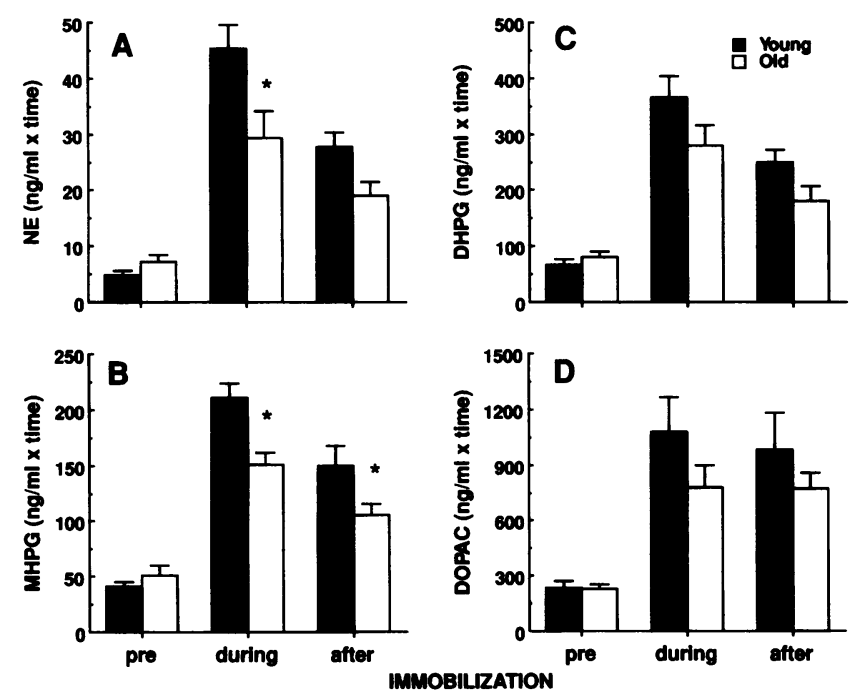

Figure 3. Time-integrated area under the curve ( AUC) of microdialysate NE $(A)$, MHPG $(B)$, DHPG $(C)$, and DOPAC $(D)$ in young and old male Fischer $344 / \mathrm{N}$ rats, before, during, and after immobilization. Preimmobilization AUCs of NE, MHPG, DHPG, and DOPAC were similar between age groups. During immobilization, the AUCs of NE ( $P$ $<0.003)$ and MHPG $(P<0.002)$ were lower in old vs young rats (asterisk). There was a nonsignificant trend towards a lower AUC of DHPG $(P<0.06)$ in old rats, whereas the AUCs for DOPAC did not vary with age. After immobilization, the AUCs of all catecholamines were lower in old rats, although the age difference was significant only for MHPG $(P<0.03)$ (asterisk). In both age groups, AUCs of all catecholamines were significantly greater after versus before immobilization (NE: young, $P<0.0006$; old, $P<0.05$; MHPG: young, $P$ $<0.0006$; old, $P<0.01$; DHPG: young, $P<0.0006$; old, $P<0.05$; DOPAC: young, $P<0.001$; old, $P<0.01$ ).
Total AUCs of DHPG, MHPG, DOPAC, and HVA were significantly larger in old rats, and total AUCs of NE, EPI, DOPA, and DA similar between groups of age (Table I). Net AUCs of EPI, and DOPA were significantly smaller, and net AUC of HVA significantly larger in old rats; the net AUCs of all other compounds were similar between groups of age (Table I).

Plasma levels of all compounds during IMMO, expressed as percent increases above pre-immobilization values, were consistently and significantly smaller in old rats (data not shown).

\section{Discussion}

In the current study, we investigated whether the responsivity of central and peripheral catecholaminergic systems to immobilization stress is altered in healthy old versus young rats. We found baseline ECF levels of NE, DHPG, MHPG, and DOPAC in the PVN, and baseline TH mRNA levels in the LC, to be similar in young and old Fischer 344/N male rats. Each of these parameters rose significantly in response to $2 \mathrm{~h}$ of IMMO in both groups of rats; however, there were significant age-related reductions in the magnitudes of IMMO-induced increases in ECF levels of catecholamines in the PVN which attained statistical significance for ECF NE and MHPG, whereas the corresponding increases in TH mRNA levels in the LC were similar between age groups. Basal levels of catecholamines in plasma were significantly higher in old rats; however, there was an age-related diminution in the plasma catecholamine response to IMMO. Taken together, our data suggest a generalized decrease in responsivity of a large array of catecholamines and their metabolites to IMMO, both in the central and peripheral catecholaminergic systems, in old versus young rats.

Sources and meaning of NE, DHPG, MHPG, and DOPAC released in the $P V N$. The PVN of the hypothalamus consists of an area of neurons containing $\mathrm{CRH}$, vasopressin, or both peptides, all terminating in the median eminence, and an area of magnocellular neurons containing almost exclusively vasopressin and terminating in the neural lobe of the pituitary (reviewed in reference 27). Given the respective dimensions of the microdialysis probe and the PVN, it would not possible to sample 

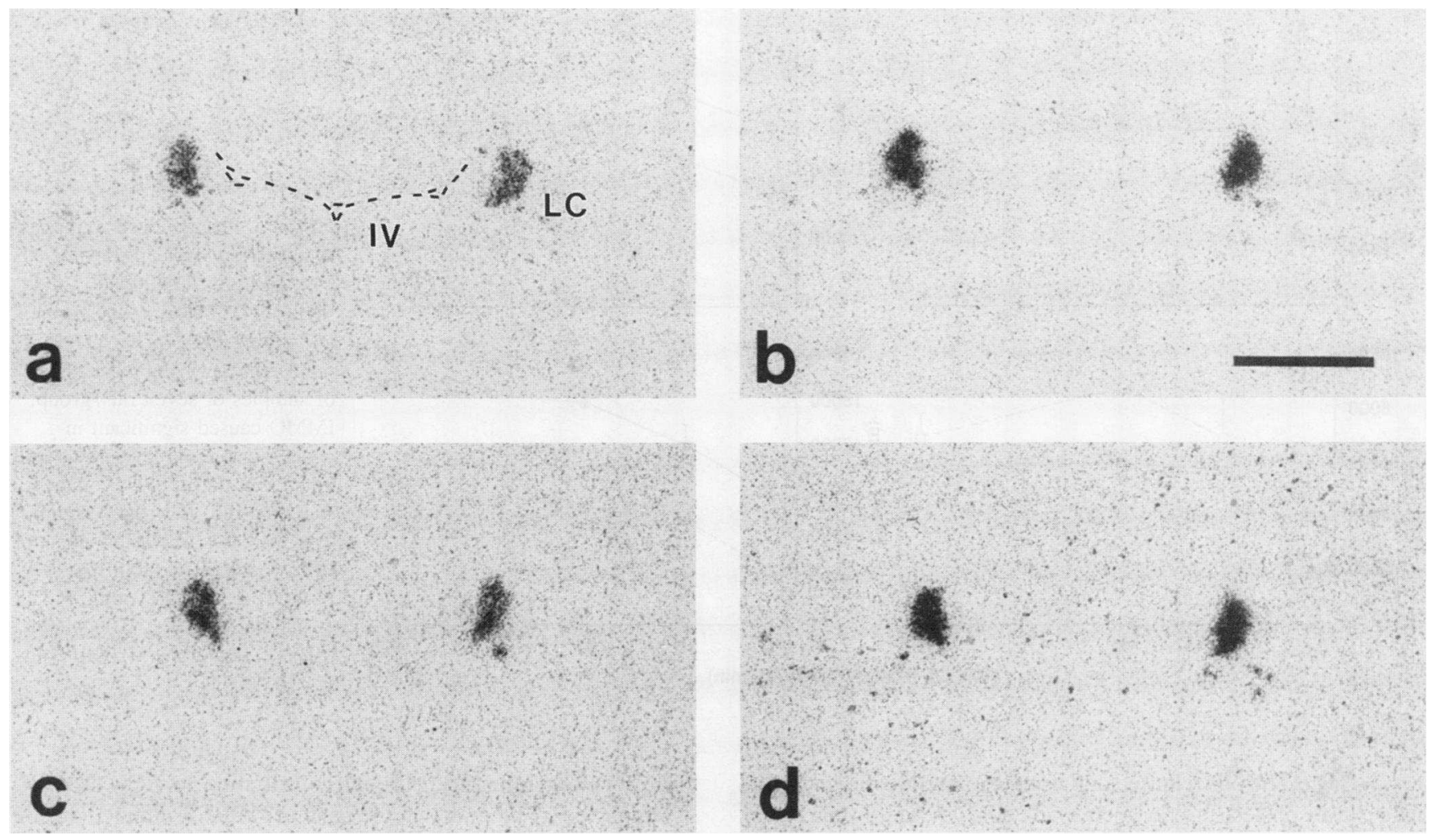

Figure 4. Effects of immobilization on tyrosine hydroxylase mRNA levels in the locus ceruleus $(L C)$ of young and old $344 / \mathrm{N}$ Fischer rats as determined by in situ hybridization: young control $(a)$, young immobilized $(b)$, old control $(c)$, old immobilized $(d)$. Bar, $1 \mathrm{~mm}$. In $a$, the Nisslstained section was projected onto the film image, and the fourth ventricle was outlined. Basal levels were similar between age groups. Immobilization produced significant increases $(P<0.0001)$ in TH mRNA levels in both groups; the increases, however, were not different between age groups. $n=6$ or 7 /group.

exclusively from any of these two areas, even with the minute probe employed in this study. Therefore, the compounds measured here were most likely derived from both parvi- and magnocellular areas. The position of the probe was verified to be within the PVN post hoc.

$N E$. Our observation of an age-related reduction in IMMOinduced increases in ECF levels of NE in the PVN of male $344 / \mathrm{N}$ Fischer rats is consistent with decreases in various parameters of noradrenergic function in the aged brain previously reported in the literature $(3-6,28)$. IMMO-induced increases in NE levels in the PVN were accompanied by similar increases in ECF levels of DHPG.

$D H P G$. DHPG, the main intraneuronal brain metabolite of $\mathrm{NE}$, derives from two sources: reuptake of $\mathrm{NE}$ released into the synaptic cleft, and net leakage of NE from intracytoplasmic vesicular stores. The relative contribution of these two processes is dependent upon the level of neuronal activity (20). Consistent with the smaller levels of NE observed in the PVN of aged rats during IMMO, we observed a trend towards reduced levels of DHPG during IMMO in aged rats.

Table I. Effects of 2 h of Immobilization on Plasma Cathecholamines and Their Metabolites in 3-4-mo old (young) and 24-mo-old (old) Cannulated Conscious 344/N Male Fischer Rats

\begin{tabular}{|c|c|c|c|c|c|c|c|c|c|}
\hline & \multicolumn{3}{|c|}{ Basal } & \multicolumn{3}{|c|}{ Total AUC } & \multicolumn{3}{|c|}{ Net AUC } \\
\hline & \multicolumn{3}{|c|}{$\mathrm{pg} / \mathrm{ml}$} & \multicolumn{3}{|c|}{$(p g / m l \times m i n) \times 100$} & \multicolumn{3}{|c|}{$(p g / m l \times \min ) \times 100$} \\
\hline & Young & Old & $\mathbf{P}$ & Young & Old & $\mathbf{P}$ & Young & Old & $\mathbf{P}$ \\
\hline $\mathrm{NE}$ & $581 \pm 147$ & $1808 \pm 574$ & N.S. & $3584 \pm 621$ & $4271 \pm 1092$ & N.S. & $2887 \pm 492$ & $2102 \pm 422$ & N.S. \\
\hline Epl & $35 \pm 7$ & $638 \pm 186$ & 0.007 & $8168 \pm 410$ & $6621 \pm 872$ & N.S. & $8126 \pm 405$ & $5855 \pm 778$ & 0.02 \\
\hline DHPG & $1051 \pm 60$ & $2895 \pm 408$ & 0.0008 & $5036 \pm 268$ & $6575 \pm 541$ & 0.02 & $3775 \pm 303$ & $3100 \pm 381$ & N.S. \\
\hline MHPG & $1910 \pm 401$ & $5980 \pm 1397$ & 0.01 & $8624 \pm 512$ & $16013 \pm 1232$ & 0.0001 & $6334 \pm 723$ & $8837 \pm 1052$ & N.S. \\
\hline DOPA & $497 \pm 32$ & $785 \pm 58$ & 0.0009 & $61251 \pm 43$ & $1246 \pm 118$ & N.S. & $655 \pm 52$ & $304 \pm 108$ & 0.01 \\
\hline DA & $58 \pm 29$ & $163 \pm 28$ & 0.03 & $419 \pm 56$ & $703 \pm 128$ & N.S. & $359 \pm 71$ & $535 \pm 99$ & N.S. \\
\hline DOPAC & $961 \pm 53$ & $2961 \pm 529$ & 0.003 & $6787 \pm 589$ & $9945 \pm 1022$ & 0.02 & $5634 \pm 617$ & $6392 \pm 646$ & N.S. \\
\hline HVA & $2100 \pm 410$ & $16430 \pm 4290$ & 0.006 & $25490 \pm 1390$ & $58790 \pm 9331$ & 0.004 & $22969 \pm 1302$ & $39070 \pm 5146$ & 0.01 \\
\hline
\end{tabular}

Values are the mean $\pm \operatorname{SEM}(n=7$ rats/group). 

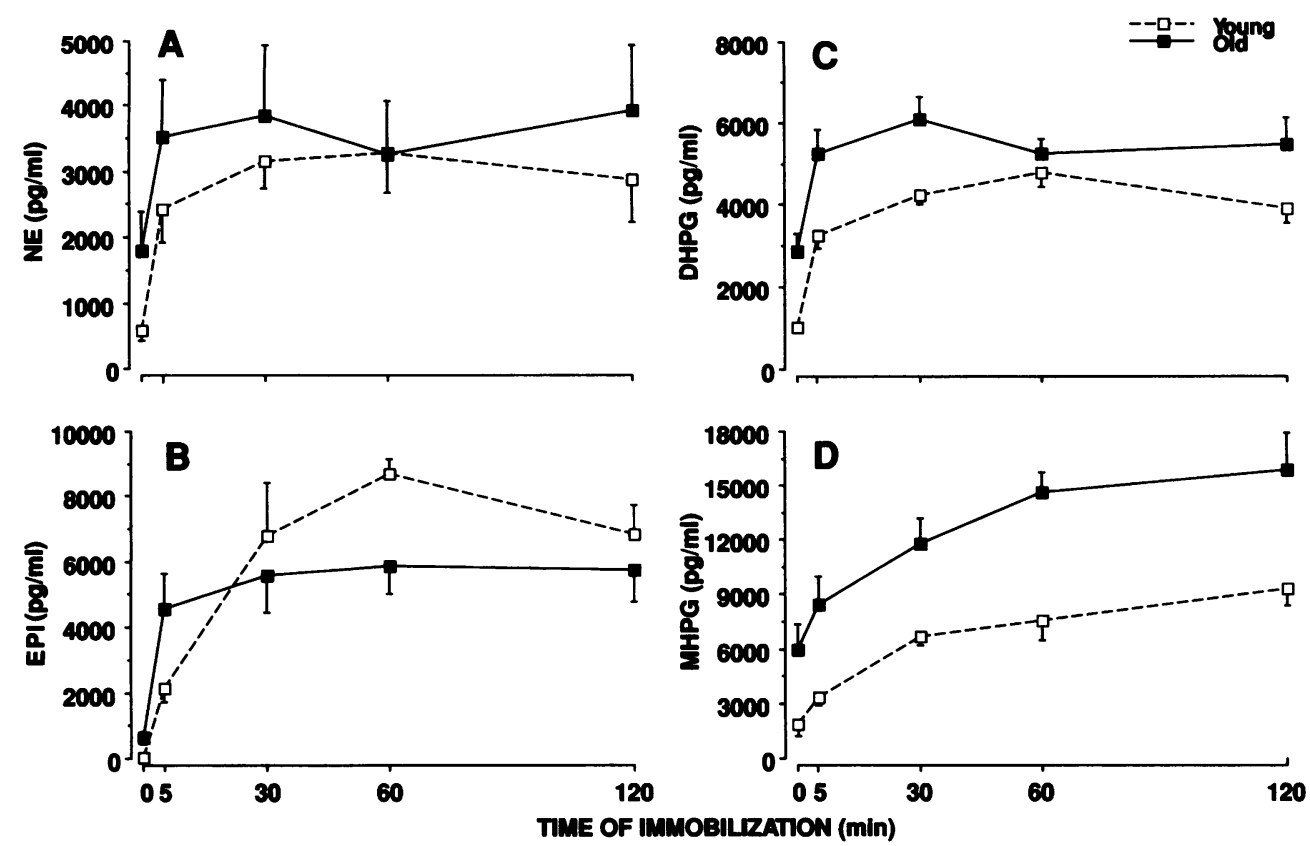

Figure 5. Plasma levels expressed as absolute values $(\mathrm{pg} / \mathrm{ml})$ of $\mathrm{NE}$ $(A)$, EPI $(B)$, DHPG $(C)$, and MHPG $(D)$ before (time zero) and during IMMO. Each timepoint represents the mean \pm SEM of samples of seven rats/group. IMMO caused significant increases in plasma levels of NE in young but not in old, (young, $P$ $<0.003$; old, $P>0.4$ ); significant increases in plasma levels of EPI (young, $P<0.0001$; old, $P$ $<0.001$ ); DHPG (young, $P$ $<0.0001$; old, $P<0.002)$ and MHPG (young $P<0.0001$; old, $P<0.0003)$.

$M H P G$. The NE released and not recaptured by nerve terminals is transported into non-neuronal cells, where it is converted by catechol- $O$-methyl transferase (COMT) to normetanephrine, and subsequently deaminated by monoamine oxidase (MAO) to MHPG (29). Another important source of MHPG is DHPG which, unlike NE, can easily cross cell membranes and be converted by COMT to MHPG (29). Responses of ECF MHPG levels were significantly smaller in old versus young rats. Taken together, our data on ECF NE, DHPG, and MHPG are compatible with decreased noradrenergic activity in the PVN of old rats during IMMO.

$D O P A C$. We found no age-associated changes in ECF levels of DOPAC at baseline, or during or after IMMO. DOPAC in the PVN has several origins as it can derive from noradrenergic terminals, dopaminergic cell bodies, or dopaminergic terminals
(20). A noradrenergic neuronal contribution to ECF DOPAC is suggested by the fact that the peak of DOPAC attained during IMMO was delayed by 60 min compared to the peak of NE.

$60-90 \%$ of the catecholamines released into the PVN originates from noradrenergic areas (i.e., A1-A3) located in the pons, and are subsequently transported to the PVN through the noradrenergic bundle $(30,31)$. The fact that IMMO elicited increases in NE and MHPG which were significantly smaller in old rats, raises two possibilities: the first is lesser stressinduced activation of the PVN via A1-A3 noradrenergic areas in the pons and the second is greater inhibition of the PVN via GABAergic or other inhibitory pathways. These possibilities are not mutually exclusive.

Resting and stress mRNA levels of TH in the LC and functional correlations between $L C$ and PVN. Consistent with the
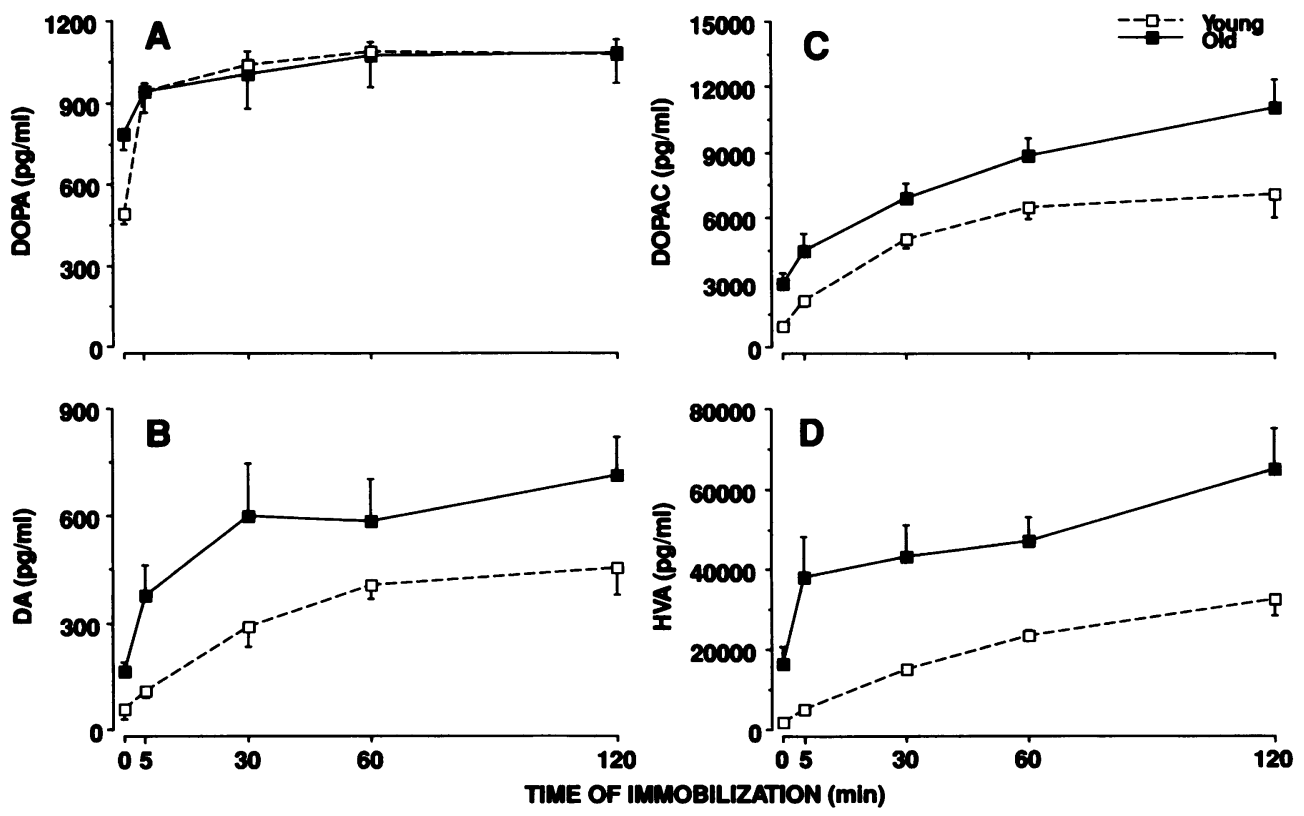

Figure 6. Plasma levels expressed as absolute values of DOPA $(A)$ DA $(B)$, DOPAC $(C)$, and HVA $(D)$ before (time zero) and during IMMO. Each time-point represents the mean \pm SEM of samples of seven rats/group. IMMO caused significant increases in plasma levels of DOPA in young but not in old (young, $P<0.0001$; old, $P>0.2$ ); significant increases in plasma levels of DA, (young, $P<0.0001$; old, $P$ $<0.01$ ) DOPAC (young, $P$ $<0.0001$; old, $P<0.0001)$ and HVA (young, $P<0.0001$; old, $P$ $<0.004)$. 
observation that the number of LC neurons does not decrease with aging in $\mathrm{F} 344 / \mathrm{N}$ rats (12), mRNA levels of $\mathrm{TH}$, the ratelimiting enzyme in catecholamine biosynthesis, were similar . between young and old rats both at baseline and after $2 \mathrm{~h}$ of IMMO. A period of $2 \mathrm{~h}$ of IMMO was long enough not only to increase TH activity, as others have reported (32), but also to activate $\mathrm{TH}$ gene expression $(33,34)$. A bidirectional relationship exists in the CNS between catecholaminergic and CRH systems (2). Thus, CRH immunoreactive fibers and receptors are present in the $\mathrm{LC}$, and $\mathrm{CRH}$, either administered into the cerebral ventricles, or endogenously secreted during stress, increases the activity of LC neurons (reviewed in reference 30 ). An important source of CRH mediating the increase of TH in the LC is represented by the PVN, although $\mathrm{CRH}$ may also derive from the amygdala and/or certain brain stem nuclei (33, 34). The LC, on the other hand, provides through the dorsal noradrenergic bundle a small portion of the noradrenergic fibers that innervate the hypothalamus (reviewed in reference 30 ). However, given the fact that this contribution is modest compared with $\mathrm{A} 1-\mathrm{A} 3$ and $\mathrm{C} 1-\mathrm{C} 3$ areas, our observation that NE levels in the PVN were lower, and TH mRNA levels in the LC similar, in old rats during stress is not surprising.

Baseline and IMMO levels of catecholamine compounds in plasma. In the current study, baseline plasma levels of NE, EPI, DHPG, MHPG, DOPA, DA, DOPAC, and HVA, as well as absolute plasma levels of most catecholamines or their metabolites during IMMO, were much higher in old versus young rats. As with hypothalamic catecholamines, responsivity of plasma catecholamines to IMMO was consistently reduced in old rats.

DOPA. DOPA, the product of the rate-limiting step in catecholamine biosynthesis via hydroxylation of tyrosine, is the precursor of DA, NE, and EPI (35). Acute changes in plasma DOPA levels may reflect activation of catecholamine biosynthesis in sympathetic nerve terminals, providing an indirect index of TH activation (25). In agreement with a prior report of increased basal TH activity in the adrenal with aging (16), we observed significantly higher basal plasma levels of DOPA in old rats. We found that IMMO significantly increased plasma DOPA in young rats, as previously reported (25), but failed to do so in old rats. In both age groups, increments in plasma DOPA were less than those of NE, confirming prior observations (36). Future studies should verify whether stress-induced activation of TH mRNA reported in the adrenal gland of young rats (37) is deficient in old rats.

$D A$. DA levels increased progressively during IMMO in both young and old rats suggesting that DOPA synthesis was being continuously activated.

DOPAC. The response of plasma DOPAC to IMMO was larger in old rat, as indicated by greater total AUC in this group of age. As plasma levels of DOPAC depend on new synthesis of DA in nerve terminals (25), our observation of larger IMMOinduced levels of DOPAC in aged rats is compatible with larger DA synthesis in old compared to young rats during IMMO.

$H V A$. Responses of plasma HVA to IMMO were also significantly greater in aged rats as indicated by both total and net AUC. As plasma HVA is the major final metabolic product of DA (25), this observation raises the possibility that the responses of the dopaminergic system to IMMO could be increased in old rats.

$N E$. Consistent with most human (14) and some (13, 38, 39) but not all (40-42) animal studies, we found significantly higher basal plasma NE levels in old versus young rats, suggesting increased basal sympathetic activity with aging. Al- though decreased metabolic clearance of NE in aged rats may also account for this finding, we are not aware of any published study on this. IMMO did not elicit significant increases in plasma NE levels in old rats as it did in young controls, probably because of a "ceiling" effect for this particular stressor. The adrenal contribution to circulating NE during IMMO takes place in the first $20 \mathrm{~min}$, when the adrenal medulla supplies about 1/ 3 of plasma NE, whereas later during IMMO, NE derives almost entirely from sympathetic neurons (43). Our data are consistent with a relative deficiency of both sources of circulating NE in old rats during IMMO.

EPI. Unlike McCarty et al. $(41,42)$ we observed an increase in baseline EPI levels, with a corresponding decrease in EPI responsivity to IMMO, in old vs young rats. Because the adrenal medulla is the major source of circulating EPI, this observation suggests that adrenal medullary activity is increased at baseline and decreased during acute IMMO in aged rats.

DHPG. Old rats exhibited significantly higher basal plasma levels of DHPG, probably due to higher levels of NE which is taken up by noradrenergic terminals and deaminated in DHPG. We observed in both age groups significant increases of DHPG, although of smaller amplitude in old rats. Because increases in plasma DHPG during sympathetic stimulation derive from NE released into the synaptic cleft and subsequently recaptured by uptake (44), our data suggest that NE after release from sympathetic nerve terminals, undergoes similar reuptake and further metabolism to DHPG in both age groups.

$M H P G$. Plasma MHPG can derive from $O$-methylation of DHPG, monoaminooxidation of normetanephrine, and $O$-methylation of NE after extraneuronal uptake (29). Absolute levels of MHPG were significantly higher in plasma of old versus young rats at baseline and during IMMO, indicating greater noradrenergic activation in old rats under basal conditions. The responsivity of MHPG to IMMO stress was decreased with age. A decreased removal rate would also account for the higher plasma MHPG levels observed in aged rats; however, the fact that during IMMO plasma levels of MHPG increased much more in young than in old rats does not support this hypothesis.

In summary, these data indicate that the responses of central and peripheral catecholaminergic systems to IMMO are decreased in aged rat. The responsiveness of the peripheral dopaminergic system to this stressor, however, seems to be in old rats either preserved or exagerrated. Whether this represents an adaptive mechanism to old age remains to be determined.

Thyroid hormones can influence the sympathetic system at various levels by regulating number of $\beta$-adrenergic receptors, and adrenergic up-regulatory mechanisms (reviewed in references 45 and 46); because $\mathrm{F} 344 / \mathrm{N}$ rats exhibit central hypothyroidism with advancing age (47), future studies should clarify whether the hypothyroidism present in this strain of rats at old age may also have contributed to the decreased catecholaminergic responsivity to IMMO stress reported in the current study.

\section{Acknowledgments}

We would like to thank Dr. Richard McCarty, University of Virginia, for his critical comments upon reviewing this manuscript.

\section{References}

1. Meites, J. 1991. Role of hypothalamic catecholamines in aging processes. Acta Endocrinol-Cop. 125:98-103.

2. Chrousos, G. P., and P. W. Gold. 1992. The concept of stress and stress system disorders. J. Am. Med. Assoc. 267:1244-1252. 
3. Estes, K. S., and J. W. Simpkins. 1980. Age-related alterations in catecholamine concentrations in discrete preoptic area and hypothalamic regions in the male rat. Brain Res. 194:556-560.

4. Ida, Y., M. Tanaka, A. Tsuda, Y. Kohno, Y. Hoaki, R. Nakagawa, K. limori, and N. Nagasaki. 1984. Recovery of stress-induced increases in noradrenaline turnover is delayed in specific brain regions of old rats. Life Sci. 34:23572363.

5. Long-Wu, Z., B. Weiss, J. S. Freilich, and L. H. Greenberg. 1984. Impaired recovery of alpha ${ }^{1}$ - and alpha ${ }^{2}$-adrenergic receptors in brain tissue of aged rats. J. Gerontol. 39:538-546.

6. Bickford, P., C. Heron, D. A. Young, G. A. Gerhardt, and R. de La Garza. 1992. Impaired acquisition of novel locomotor tasks in aged and norepinephrinedepleted F344 rats. Neurobiol. Aging. 13:475-481.

7. Meites, J. 1990. Aging: hypothalamic catecholamines, neuroendocrineimmune interactions, and dietary restriction. PSEBM (Proc.Soc. Exp. Biol. Med.). 195:304-311.

. 8. Olpe, H. R., and M. W. Steinmann. 1982. Age-related decline in the activity of noradrenergic neurons of the rat locus ceruleus. Brain Res. 251:174-176.

9. Sturrock, R. R., and K. A. Rao. 1985. A quantitative histological study of neuronal loss from the locus ceruleus of ageing mice. Neuropathol. Appl. Neurobiol. 11:55-60.

10. Chan-Palay, V., and E. Asan. 1989. Quantitation of catecholamine neurons in the locus ceruleus in human brains of normal young and older adults and in depression. J. Comp. Neurol. 287:357-372.

11. Tomonaga, M. 1983. Neuropathology of the locus ceruleus: a semi-quantitative study. J. Neurol. 230:231-240.

12. Goldman, G., and P. D. Coleman. 1981. Neuron numbers in locus ceruleus do not change with age in Fischer 344 rat. Neurobiol. Aging. 2:33-36.

13. Michalikova, S., H. Balazova, D. Jezova, and R. Kvetnansky. 1990 Changes in circulating catecholamine levels in old rats under basal conditions and during stress. Bratisl. Lek. Listy. 91:689-693.

14. Ziegler, M. G., C. R. Lake, and I. J. Kopin. 1976. Plasma noradrenaline increases with age. Nature (Lond.) 261:333.

15. Garty, M., R. Stull, I. J. Kopin, and D. S. Goldstein. 1989. Skin color, aging, and plasma L-dopa levels. J. Auton. Nerv. Syst. 26:261-263.

16. Kvetnansky, R., E. Jahnova, T. Torda, V. Strbak, V. Balaz, and L. Macho 1978. Changes of adrenal catecholamines and their synthesizing enzymes during ontogenesis and aging in rats. Mech. Ageing Dev. 7:209-216.

17. Banerji, T. K., T. A. Parkening, and T. J. Collins. 1984. Adrenomedullary catecholaminergic activity increases with age in male laboratory rodents. J. Ger ontol. 39:264-268.

18. Roth, G. S. 1990. Hormone/neurotransmitter action during aging: the calcium hypothesis of impaired signal transduction. Rev. Biol. Research Aging. 4:243-252.

19. P. J. Scarpace. 1988. Decreased receptor activation with age. J. Am. Geriatr. Soc. 36:1067-1071.

20. Pacak, K., I. Armando, K. Fukuhara, R. Kvetnansky, M. Palkovits, I. J. Kopin, and D. S. Goldstein. 1992. Noradrenergic activation in the paraventricular nucleus during acute and chronic immobilization stress in rats: an in vivo microdialysis study. Brain Res. 589:91-96.

21. Cizza, G., R. Kvetnansky, M. E. Tartaglia, M. R. Blackman, G. P. Chrousos, and P. W. Gold. 1993. Immobilization rapidly decreases hypothalamic corticotropin-releasing hormone secretion in vitro. Life Sci. 53:233-240.

22. Paxinos, G., C. Watson. 1986. The rat brain in stereotaxic coordinates. Academic Press, San Diego, CA

23. Grima, B., A. Lamoroux, F. Blanot, N. F. Biquet, and J. Mallet. 1985 Complete coding sequence of rat tyrosine hydroxylase mRNA. Proc. Natl. Acad. Sci. USA. 82:617-621

24. Cizza, G., A. E. Calogero, L. S. Brady, G. Bagdy, E. Bergamini, M. R. Blackman, G. P. Chrousos, and P. W. Gold. 1994. Male Fischer 344/N rats show a progressive central impairment of the hypothalamic-pituitary-adrenal axis with advancing age. Endocrinology. 134:1611-1620.

25. Kvetnansky, R., I. Armando, V. K. Weise, C. Holmes, K. Fukuhara, A. Deka-Starosta, I. J. Kopin, and D. S. Goldstein. 1992. Plasma DOPA responses during stress: dependence on sympathoneural activity and tyrosine hydroxylation.

J. Pharmacol. Exp. Ther. 261:899-909.

26. Kvetnansky, R., C. L. Sun, C. R. Lake, N. B. Thoa, T. Torda, and I. J. Kopin. 1978. Effects of handling and forced immobilization on rat plasma levels of epinephrine, norepinephrine, and dopamine $\beta$-hydroxylase. Endocrinology. 103:1868-1874

27. Cizza, G., and E. M. Sternberg. 1994. The role of the hypothalamicpituitary-adrenal in the susceptibility to autoimmune/inflammatory disease. Immunomethods. 5:73-78.

28. McIntosh, H. H., and T. C. Westfall. 1987. Influence of aging on catecholamine levels, accumulation, and release in F-344 rats. Neurobiol. Aging. 8:233239.

29. Eisenhofer, G., D. S. Goldstein, T. G. Ropchak, H. Q. Nguyen, H. R. Keiser, and I. J. Kopin. 1988. Source and physiological significance of plasma 3,4-dihydroxyphenylglycol and 3-methoxy-4-hydroxyphenylglycol. J. Autonom. Nerv. Syst. 24:1-14.

30. Mezey, E., and M. Palkovits. 1991. CRF-containing neurons in the hypothalamic paraventricular nucleus: regulation, especially by catecholamines. Front. Neuroendocrin. 12:23-37.

31. Ceccatelli, S., R. Cortes, and T. Hokfelt. 1991. Effect of reserpine and colchicine on neuropeptide mRNA levels in the rat hypothalamic paraventricular nucleus. Mol. Brain Res. 9:57-69.

32. Zigmond, R. E., M. A. Schwarzschild, and A. R. Rittenhouse. 1989. Acute regulation of tyrosine hydroxylase by nerve activity and by neurotransmitters via phosporylation. Ann. Rev. Neurosci. 12:415-461.

33. Mamalaki, E., R. Kvetnansky, L. S. Brady, P. W. Gold, and M. Herkenham. 1992. Repeated immobilization stress alters tyrosine hydroxylase, corticotropin-releasing-hormone and corticosteroid receptor messenger ribonucleic acid levels in rat brain. $J$. Neuroendocrinol. 4:689-699.

34. Smith, M., L. Brady, J. Glowa, P. W. Gold, and M. Herkenham. 1991. Effect of stress on tyrosine hydroxylase mRNA levels in the locus ceruleus by in situ hybridization. Brain Res. 544:26-32.

35. Kopin, I. J. 1985. Catecholamine metabolism: basic aspects and clinical significance. Pharmacol. Rev. 37:333-364.

36. Goldstein, D. S., R. Udelsman, G. Eisenhofer, R. Stull, H. R. Keiser, and I. J. Kopin with the technical assistance of C. J. Folio. 1987. Neuronal sources of plasma dihidroxyphenylalanine. J. Clin. Endocr. Metab. 64:856-861.

37. McMahon, A., R. Kvetnansky, K. Fukuhara, V. K. Weise, I. J. Kopin, and E. L. Sabban. 1992. Regulation of tyrosine hydroxylase and dopamine $\beta$ hydroxylase mRNA levels in rat adrenals by a single and repeated immobilization stress. J. Neurochem. 58:2124-2130.

38. Irwin, M., R. Hauger, and M. Brown. 1992. Central corticotropin-releasing-hormone activates the sympathetic nervous system and reduces immune function: increased responsivity of the aged rat. Endocrinology. 131:1047-1053.

39. Chiueh, C. C., S. M. Nespor, and S. I. Rapoport. 1980. Cardiovascular, sympathetic, and adrenal cortical responsiveness of aged Fischer 344 rats to stress. Neurobiol. Aging. 1:157-163.

40. Avakian, E. V., and S. M. Horvath. 1982. Influence of aging and tyrosine hydroxylase inhibition on tissue levels of norepinephrine during stress. J. Gerontol. 37:257-261.

41. McCarty, R. 1984. Effects of 2-deoxyglucose on plasma catecholamines in adult and aged rats. Neurobiol. Aging. 5:285-289.

42. McCarty, R. 1985. Sympathetic-adrenal medullary and cardiovascula responses to acute cold stress in adult and aged rats. J. Autonom. Nerv. Syst. 12:15-22.

43. Kvetnansky, R., V. K. Weise, N. B. Thoa, and I. J. Kopin. 1979. Effects of chronic guanethidine treatment and adrenal medullectomy on plasma levels of catecholamines and corticosterone in forcibly immobilized rats. J. Pharmacol. Exp. Ther. 209:287-291.

44. Goldstein, D. S., G. Eisenhofer, R. Stull, C. J. Folio, H. R. Keiser, and I. J. Kopin. 1988. Plasma dihydroxyphenylglycol and the intraneuronal disposition of norepinephrine in humans. J. Clin. Invest. 81:231-220.

45. Silva, J. E., and L. Landsberg. 1991. Catecholamines and the sympathoadrenal system in hypothyroidism. In Werner and Ingbar's The Thyroid. L. E. Braverman and R. D. Utiger, editors. J. B. Lippincott Philadelphia, New York, London, Hagerstown.

46. Whybrow, P. C., and A. J. Prange. 1981. A hypothesis of thyroid-catecholamine-receptor interaction. Arch. Gen. Psychiat. 38:106-113.

47. Cizza, G., L. S. Brady, A. E. Calogero, G. Bagdy, A. B. Lynn M. A. Kling, M. R. Blackman, G. P. Chrousos, and P. W. Gold. 1992. Central hypothyroidism is associated with advanced age in male $344 / \mathrm{N}$ rats: in vivo and in vitro studies. Endocrinology. 131:2672-2680. 